\title{
Lighting Analysis and Texture Modification of 3D Human Face Scans
}

\author{
Xiaozheng Zhang, Sanqiang Zhao and Yongsheng Gao \\ Computer Vision and Image Processing Laboratory, \\ Griffith School of Engineering, Griffith University, \\ 170 Kessels Road, Nathan Qld 4111, Australia \\ \{x.zhang, s.zhao,yongsheng.gao\}@griffith.edu.au
}

\begin{abstract}
The $3 D$ scanning of human faces is an important tool to acquire accurate shape and texture information of heads and faces for both animation and recognition purposes. One of the most popular scanning tools is Cyberware head and face $3 D$ colour scanner. Currently, 3D face databases scanned using the Cyberware head and face $3 D$ colour scanner databases are publicly available and have been used in many face recognition system as the training data, one of which is USF human ID 3-D database. The mechanism of facial texture capturing, however, remains unclear due to the special settings of the light sources during scanning. The direct application of the acquired texture intensity as the albedo of the face surfaces leads to suboptimal modelling results. This paper analyses the lighting configuration of the scanner and proposes an appropriate reflectance model to describe the reflecting mechanism when scanning. The texture modifications can be carried on in the existing scanning results without any further scans. Experiment results show that the proposed reflectance model can better approximate the reflecting situation of scanning so that the acquired textures can be directly used as diffuse reflectance coefficients and achieve better rendering results.
\end{abstract}

Key words: $3 D$ face model, $3 D$ scanner, facial textures, lighting condition, reflection model.

\section{Introduction}

The 3D scanner (e.g., Cyberware head and face 3D colour scanner [9]) provides accurate 3D shape information of human faces. While capturing shape information by a laser beam, the head of the subject is also illuminated by movable light sources installed on the scanner's head for the camera to capture a texture map of the face surface. The synchronisation of shape and texture acquisition is critical so that the point-wise correspondence could be established during scanning. For the Cyberware head and face 3D colour scanner, white light sources are then installed on the scanning head near the 3D camera. During scanning, the region being scanned for shape information is immediately illuminated by the lamp and captured for texture information. In this way, both shape and texture information of the same points on the face surface is measured at almost the same time to prevent the movement of the subject and the consequent misalignment.

Then, the shape and texture information can be used in various applications such as re-rendering under new illumination and viewing conditions. In computer vision and pattern recognition, they are also used as discriminating features for human face recognisers to perform face recognition. Since the light source in the scanner is movable, the lighting condition for different points on the surface may be different. Consequently, the direct texture-mapping of the original captured image intensity to the object shape cannot synthesise correct 2D images under ordinary fixed light source(s). In computer vision, they are not correctly extracted from the subject to reflect genuine intrinsic features for classifications. This paper proposes a new lighting model to address this texture adjustment and reestimation problem. Under Lambertian reflectance model, the captured texture map can be adjusted using the proposed texture modification scheme to resemble Lambertian diffuse reflectance coefficient map (i.e., bidirectional reflectance distribution function, BRDF). 


\section{The reflectance model for the 3D scanning}

This paper is motivated by the use of a large dataset of face scans in the synthesis of 3D faces [2] and face recognition across variation in pose [3]. In these studies, 200 3D face scans were used as prior knowledge of human faces for building a 3D morphable face model based on principle component analysis and for establishing a set of intrinsic parameters for recognition. Both of the geometric and textural information are acquired using the Cyberware scanner. The scanned texture information was directly stored in the dataset as the training data for the principle component analysis. Then the principle components of facial textures were extracted and used in Equation 6 of [2] as the diffuse reflectance coefficients of Phong model [7].

Vetter and Blanz [8] explained the use of scanned texture as diffuse reflectance coefficient that the movable light source can be approximated as background lights uniformly distributed in the environment and the diffuse reflectance coefficient and the ambient reflectance coefficient were interchangeable in Lambertian model. The substitution of ambient reflectance coefficient by diffuse reflectance coefficient is valid under convexity assumption. The approximation of the movable light source of the Cyberware scanner to be ambient light source, however, is far from reality, since the light source is slim lamps which is incapable to provide a large area light and hence cannot be approximated as a uniformly distributed background light.

This paper carefully examines the lighting conditions of the scanning process of human faces for the Cyberware scanner and proposes a novel and specific lighting model to resemble the scanning condition. Using this lighting model, the textures of scanned human faces can be modified and then used as diffuse reflectance coefficients as was done in [2] and [3]. The proposed texture modification scheme considers the relationship of incident light sources and the surface geometries during scanning and then is more appropriate than the rough approximation of scanner's lights as ambient light.

\subsection{Reflectance models}

The reflected intensity (i.e., the image intensity) in Phong reflectance model is expressed as

$$
I_{r}=\left(I_{a}+I_{d} \cdot\left(\vec{N} \cdot \vec{L}_{d}\right)\right) k_{d}+I_{d} k_{s}(\vec{R} \cdot \vec{V})^{v},
$$

where $I_{a}$ and $I_{d}$ are the ambient and directional light intensities respectively, $\vec{N}$ is the surface normal direction, $\vec{L}_{d}$ is the direction of the directional light $I_{d}, k_{d}$ and $k_{s}$ are the diffuse and specular reflectance coefficients respectively, $\vec{R}$ is the reflecting direction which can be expressed as

$$
\vec{R}=2\left(\vec{N} \cdot \vec{L}_{d}\right)-\vec{L},
$$

and $\vec{V}$ is the viewing direction pointing from the surface point to the camera, and $v$ is the specular reflectance exponent.

If the surface is dull matte, it doesn't have specular reflection of the incident lights. The reflection can be approximated well by Lambertian model as

$$
I_{r}=I_{d} \cdot\left(\vec{N} \cdot \vec{L}_{d}\right) k_{d} .
$$

In the above equation, ambient light and reflection is also neglected. The ambient light is often approximated as uniformly distributed incident area light from the environment and the reflection of ambient light (i.e., ambient reflection) can be modelled using an ambient reflectance coefficient $k_{a}$ as

$$
I_{r, a m b}=I_{a} k_{a} .
$$

Under Lambertian model, $k_{a}$ can be substituted by $k_{d}$ for convex objects.

Although Lambertian model is not a perfect model for human skin, it is widely used due to the simplistic form of expressions in computer graphics and computer vision including face animation and recognition such as in $[1,5,6]$. In this research, human faces are assumed to be Lambertian surfaces as was done in [2] and [3]. The modelling of human skins as non-Lambertian surfaces will be an interesting problem, since human faces do reflect specularly. The extraction of specular reflectance coefficients may require additional lighting and acquisition equipments during the scanning process and may not be incorporated into the existing datasets.

\subsection{Analysis of the lighting conditions}

Cyberware Head \& Face Colour 3D Scanner is widely used for acquisition of the shape and texture information of 3D human faces. While scanning, the scanner projects low-intensity laser beams to the face surface. Two cameras captures the laser profile in two different viewpoints and the shape information can then be estimated using stereo vision. At the same time, 
lamps installed in the scanner's head provide light sources for texture acquisition (see the two lamps on the moving scanner head in Figure 1.)

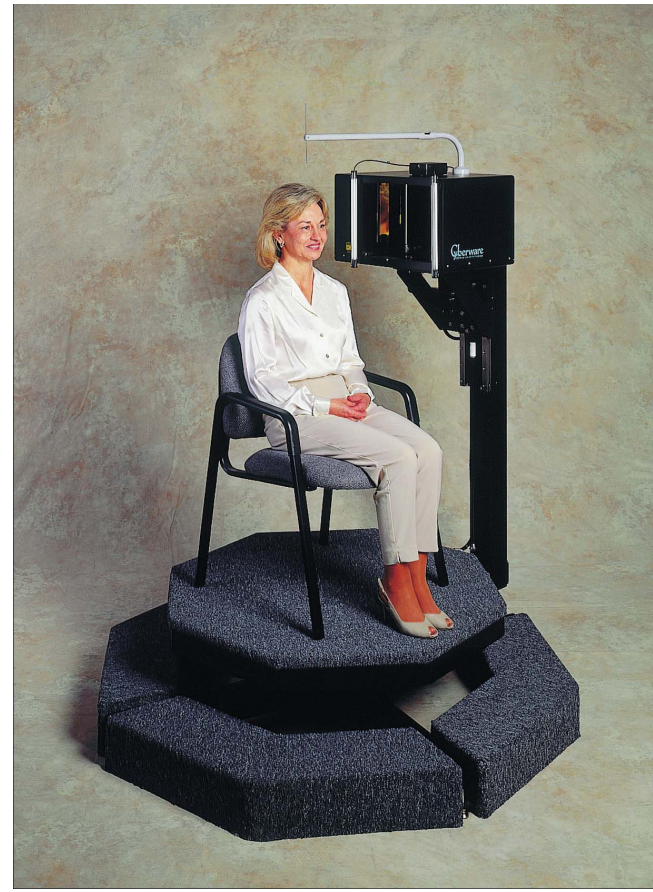

Fig. 1. The Cyberware Head \& Face Colour 3D Scanner [9] and the scanning process. Two of the lamps are installed on the scanner's head to provide light sources for texture acquisition.

Since the light source is movable, it generates a special lighting condition which is different to the existing lighting condition with fixed light sources. In $[2,3]$, this light source is approximated as ambient light, which generalised the two slim lights as the spatially uniform distributed area light within the whole hemisphere. Then the diffuse reflectance coefficient (albedo) $k_{d}$ which is equivalent to ambient reflectance coefficient $k_{a}$ can be estimated using Equation 3.

This rough assumption, however, results in suboptimal acquired facial texture evident that the regions with fine structures are relatively darker than the smoother region. To correct this texture acquisition, we propose a better lighting model for the head and face scanner, which is to improve the texture acquisition. In this paper, the incident light is modelled as movable light sources from infinity as shown in
Figure 2. While scanning the surface point $P$, the scanner camera is located in the line connecting the centre point and $P$. The lamps are located in the right and left sides of the scanner's head symmetrically and let $\alpha$ denote the angle between the incident light and the centre line (note this direction is not necessarily the normal direction).

As the heights on the human face are variable due to different facial components (e.g., the nose tip is higher than the lips), $\alpha$ is not constant. Provided the scanned surface shape and the configuration of the scanner, it is possible to calculated exact $\alpha$ values for every point. In this paper, however, we assume a constant $\alpha$ value to simplify the texture analysis since the variations of face shape from a cylinder are relatively small compared to the distance of the two lamps to the head.

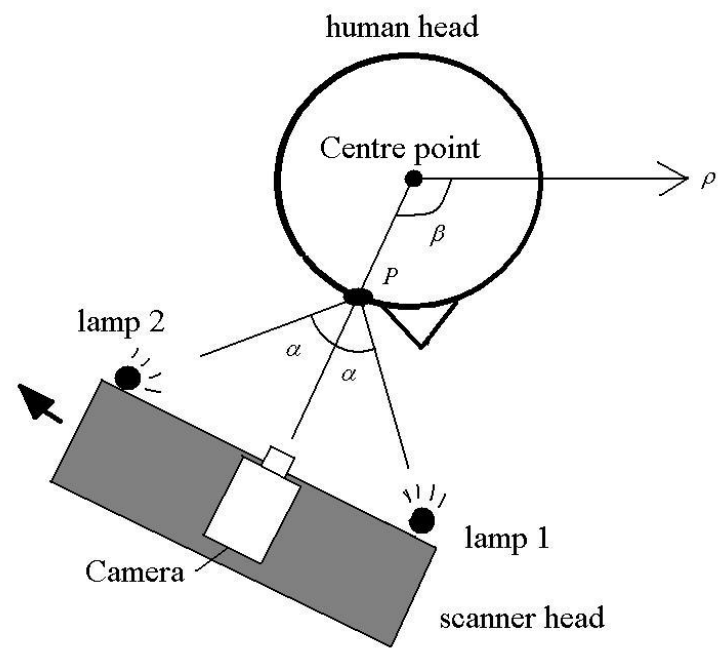

Fig. 2. The human face scanning using Cyberware Head \& Face Colour 3D Scanner.

Under Lambertian assumption, then, the acquired texture intensity can be expressed as

$$
I_{r}=I_{d} \cdot\left[\left(\vec{N} \cdot \vec{L}_{1}\right)+\left(\vec{N} \cdot \vec{L}_{2}\right)\right] \cdot k_{d},
$$

where $\vec{L}_{1}$ and $\vec{L}_{2}$ are the directions from the two lamps respectively. Suppose the angles between the two lamps and the normal direction $\vec{N}$ are $\gamma_{1}$ and $\gamma_{2}$, respectively. We have

and

$$
\cos \gamma_{1}=\left(\vec{N} \cdot \vec{L}_{1}\right),
$$




$$
\cos \gamma_{2}=\left(\vec{N} \cdot \vec{L}_{2}\right)
$$

Surface normal $\vec{N}$ is calculated using B-spline surface approximation on 16 neighbour points in a facial area as

$$
\vec{N}=\frac{\partial}{\partial x} Q(x, y) \times \frac{\partial}{\partial y} Q(x, y),
$$

where $Q(x, y)$ is the B-spline surface matrix [4]. Suppose the azimuth and zenith angles between surface normal $\vec{N}$ and centre line are $\theta$ and $\phi$ respectively, the angles $\gamma_{1}$ and $\gamma_{2}$ can be expressed as

$$
\cos \gamma_{1}=\frac{\cos \phi \cos 2(\alpha-\theta)}{\cos (\alpha-\theta)}
$$

and

$$
\cos \gamma_{2}=\frac{\cos \phi \cos 2(\alpha+\theta)}{\cos (\alpha+\theta)}
$$

Therefore, Equation 5 becomes

$$
I_{r}=I_{d} \cos \phi\left(\frac{\cos 2(\alpha-\theta)}{\cos (\alpha-\theta)}+\frac{\cos 2(\alpha+\theta)}{\cos (\alpha+\theta)}\right) k_{d} \text {. }
$$

Then the acquired texture should be modified by dividing by a factor

$$
\cos \phi\left(\frac{\cos 2(\alpha-\theta)}{\cos (\alpha-\theta)}+\frac{\cos 2(\alpha+\theta)}{\cos (\alpha+\theta)}\right)
$$

to better resemble diffuse reflectance coefficients.

The modified texture $T^{\prime}$ can then be calculated as

$$
T^{\prime}=\frac{T}{\cos \phi \cdot\left(\frac{\cos 2(\alpha-\theta)}{\cos (\alpha-\theta)}+\frac{\cos 2(\alpha+\theta)}{\cos (\alpha+\theta)}\right)},
$$

where $T$ is the original acquired texture using the scanner.

\section{Texture modifications and results}

In the experiment, we set $\alpha=30^{\circ}$. The USF human ID 3-D database was processed with the calculated shape and normal information and proposed texture modification (Equation 13). The individual facial textures were modified using Equation 10 and the results are shown in Figure 3 and 4. The IDs of these two faces in the database are 03513 and 03721 , respectively.
The average texture can also be modified in the same way since it is a linear principle component analysis. The results are shown in Figure 5 and 6.

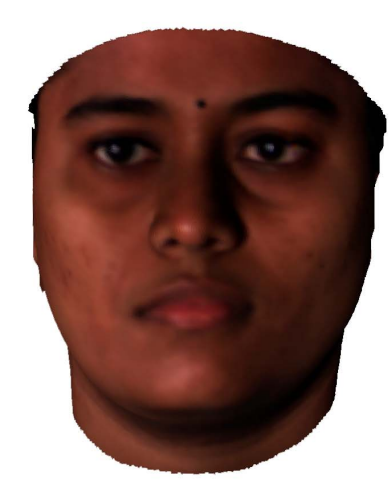

(a)

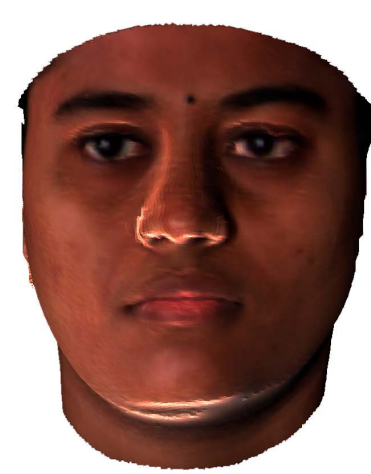

(b)
Fig. 3. The shape and texture of person 03513 in the USF human ID 3-D database (a) before texture modification, and (b) after the proposed texture modification.

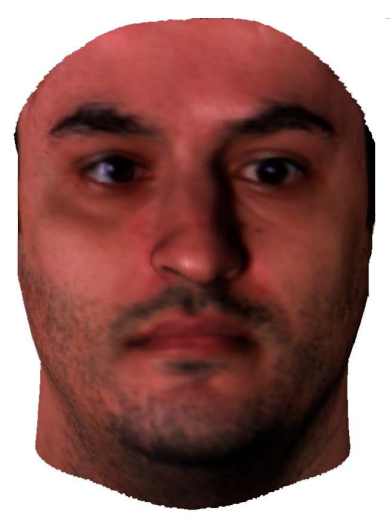

(a)

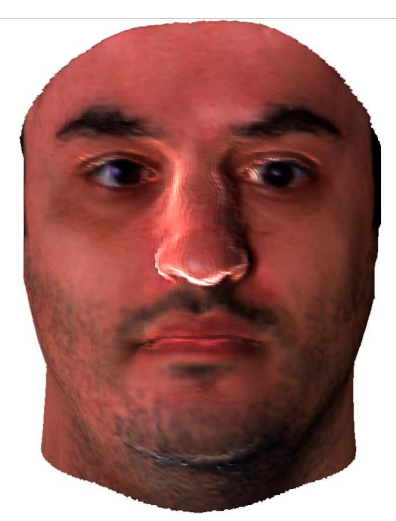

(b)
Fig. 4. The shape and texture of person 03721 in the USF human ID 3-D database (a) before texture modification, and (b) after the proposed texture modification. 


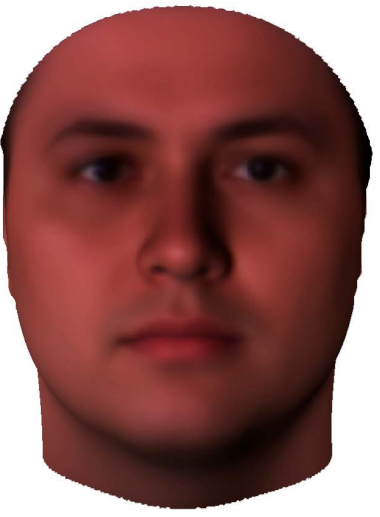

(a)

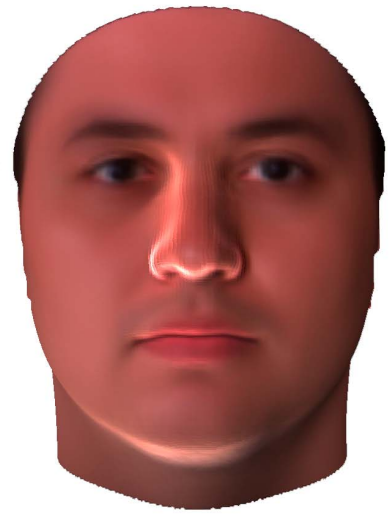

(b)
Fig. 5. The average face shape and texture in the frontal view (a) before texture modification, and (b) after the proposed texture modification.

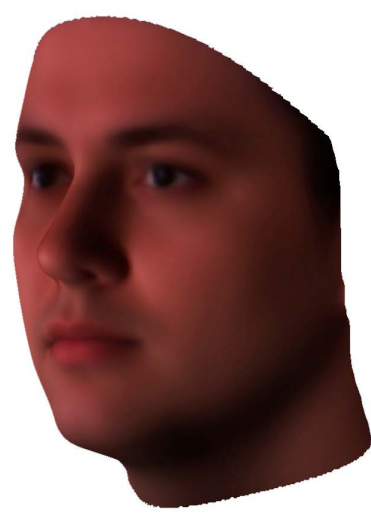

(a)

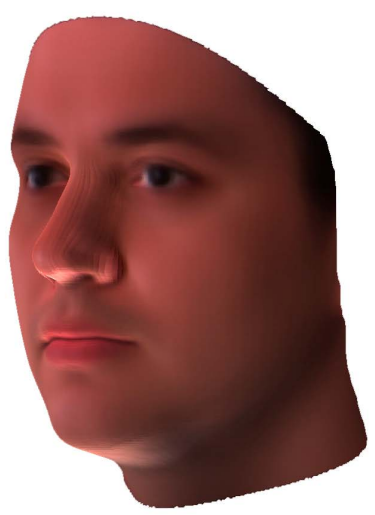

(b)
Fig. 6. The average face shape and texture from a rotated viewing angle (a) before texture modification, and (b) after the proposed texture modification.

From the above texture modification results, the modified texture can be used as the diffuse reflectance coefficient in the 3D face models while the originally acquired textures are suboptimal due to the inaccurate modelling of the lighting conditions of the scanning environment. Especially on the occluded region of the faces such as the region near eyes, the original textures tend to be dark due to the lack of consideration of the structure and the big angle between normal direction and lighting directions. The modified textures are then corrected and there are also few shadow effects on the surfaces. The texture modification approach could be used in later face recognition and face modelling process and a better result could be expected because of the validity of lighting condition approximation.

\section{Conclusion}

Cyberware Head and Face Colour 3D Scanner is one of the most widely used 3D scanners for modelling 3D face shapes and textures in the face animation and recognition research and application community. Through careful examination of the lighting condition of the scanning process of the Cyberware Head and Face Colour 3D Scanner, this paper proposed a novel lighting model and a texture modification scheme to improve the acquired texture information so that it can be used as diffuse reflectance coefficient under Lambertian reflectance model. The existing modelling of the lighting condition as ambient light is not correct due to the presence of two slim lamps in the scanner's head. The proposed light model considers the positions and moving conditions of the two lamps and therefore better resembles the scanning process. Then a modification scheme is proposed based on the new light model under Lambertian reflection model.

In experiment, 3D face models in public available database were processed and their textures were modified using the proposed approach. The experiment result shows that the proposed approach improves the acquisition of textures for the scanner and the use of this approach could subsequently improve the performance of face recognition and modelling algorithms which use the 3D face database. The future work of this research may lie in the incorporating nonLambertian reflectance models into the texture acquisition and/or modification, which could enable the estimation of both diffuse and specular reflectance coefficients of the face surface during scanning.

\section{References}

[1] D. Beymer and T. Poggio, "Face recognition from one example view," Proceedings of International Conference on Computer Vision, pp. 500-507, Cambridge, MA, USA, 1995.

[2] V. Blanz and T. Vetter, "A morphable model for the synthesis of 3D faces," Proceedings of SIGGRAPH 99 (Computer Graphics), pp. 187-194, New York, NY, USA, 1999.

[3] V. Blanz and T. Vetter, "Face recognition based on fitting a 3D morphable model," IEEE Transactions on Pattern Analysis and Machine Intelligence, vol. 25, pp. 1063-1074, 2003. 
[4] J. D. Foley, A. van Dam, S. K. Feiner, and J. F. Hughes, Computer graphics: principles and practice, 2nd ed. AddisonWesley Publishing Company, Inc., 1995.

[5] A. S. Georghiades, P. N. Belhumeur, and D. J. Kriegman, "From few to many: Illumination cone models for face recognition under variable lighting and pose," IEEE Transactions on Pattern Analysis and Machine Intelligence, vol. 23, pp. 643-660, 2001.

[6] R. Gross, I. Matthews, and S. Baker, "Appearance-based face recognition and light-fields," IEEE Transactions on Pattern Analysis and Machine Intelligence, vol. 26, pp. 449465, 2004.
[7] B. T. Phong, "Illumination for computer generated pictures," Communications of the ACM, vol. 18, pp. 311$317,1975$.

[8] T. Vetter and V. Blanz, "Estimating coloured 3D face models from single images: An example based approach," Proceedings of Computer Vision - ECCV, Freiburg, Germany, 1998.

[9] Cyberware, "Head \& Face Color 3D Scanner, " http://www.cyberware.com/products/scanners/ps.html, accessed on June 23, 2007. 